\title{
Protective effect of Shenfu injection preconditioning on lung ischemia-reperfusion injury
}

\author{
HONG ZHANG $^{1}$, ZHANHAI WAN ${ }^{1}$, XIANG YAN ${ }^{2}$, DE-GUI WANG ${ }^{3}$, YUFANG LENG ${ }^{1}$, \\ YONGQIANG LIU $^{1}$, YAN ZHANG ${ }^{1}$, HAIJUN ZHANG ${ }^{1}$ and XUENA HAN ${ }^{1}$ \\ Departments of ${ }^{1}$ Anesthesiology and ${ }^{2}$ Gerontology, The First Hospital of Lanzhou University; ${ }^{3}$ Department of \\ Anatomy and Histology, Lanzhou University School of Medicine, Lanzhou, Gansu 730000, P.R. China
}

Received May 2, 2015; Accepted April 5, 2016

DOI: $10.3892 / \mathrm{etm} .2016 .3549$

\begin{abstract}
Lung ischemia-reperfusion injury remains a problem in thoracic surgery, as minimal progress has been made concerning its prevention and control. In the present study, the protective effects and the underlying mechanism of Shenfu injection preconditioning on a rat lung ischemia-reperfusion model was investigated. Shenfu injection is a well-known Chinese traditional medicine, which is composed of Red Radix Ginseng and Radix Aconitum carmichaelii, with ginseng saponin and aconitum alkaloids as the active ingredients. A total of 72 specific pathogen-free, healthy male Wistar rats were randomly divided into control, model and Shenfu injection (10 ml/kg injection prior to injury) groups and were assessed at the following points: Ischemia $45 \mathrm{~min}$; reperfusion $60 \mathrm{~min}$; and reperfusion $120 \mathrm{~min}$. Blood collected from the aorta abdominalis was cryopreserved at $-70^{\circ} \mathrm{C}$ for the analysis of malondialdehyde (MDA) and superoxide dismutase (SOD) activity. Lung tissues were divided into three equal sections in order to assess the wet-to-dry (W/D) lung ratio, tumor necrosis factor (TNF)- $\alpha$ expression levels, myeloperoxidase (MPO) activity, alveolar damage, total protein and hematoxylin and eosin staining. The results demonstrated that the lung W/D weight ratio, TNF- $\alpha$ expression levels and SOD activity in the Shenfu group were significantly lower at $120 \mathrm{~min}$ reperfusion $(\mathrm{P}<0.05)$, as compared with the model group. MPO and MDA activity significantly decreased following reperfusion at 60 and 120 min $(\mathrm{P}<0.05)$, as compared with the model group. In addition, the degree of alveolar damage in the Shenfu group was significantly decreased $(\mathrm{P}<0.05)$, as compared with the model group. In addition, compared with the model group, the degree of alveolar damage in the Shenfu group was significantly lower $(\mathrm{P}<0.05)$; however, no significant changes
\end{abstract}

Correspondence to: Dr Xiang Yan, Department of Gerontology, The First Hospital of Lanzhou University, 1 Donggang West Road, Lanzhou, Gansu 730000, P.R. China

E-mail: zhangh12@lzu.edu.cn

Key words: lung ischemia-reperfusion injury, protective effect, Shenfu injection in total protein were observed. The extent of alveolar structural damage and the proportion of interstitial neutrophils and alveolar and interstitial red blood cells were lower in the Shenfu group, as compared with the model and control groups. Therefore, the results of the present study suggested that Shenfu injection may have protective effects on lung ischemia-reperfusion injury.

\section{Introduction}

Cell metabolic disorders and structural damage are aggravated in the viscera when the blood flow blocking is removed and the reperfusion recovered. This damage further aggravates ischemic injury following blood reperfusion, and is known as ischemia-reperfusion injury The mechanism underlying ischemia-reperfusion injury in the lungs remains controversial; however, the majority of previous studies (1-3) agree with the following statements: At the early stage of lung ischemia-reperfusion injury, a number of precursor proteins in vascular endothelial cells are activated and these cells release various cell adhesion factors, promote neutrophil adhesion and aggregation, and platelet deposition, and subsequently induce microvascular congestion. With continued reperfusion, the expression of cell adhesion molecules on the surface of neutrophils and endothelial cells is increased. Subsequently, the cells adhere and release chemotactic factors, such as tumor necrosis factor (TNF), which increase cell adhesion, promote the narrowing of capillaries and block blood perfusion (4). As various vascular systolic materials are released, the microvascular function is altered, resulting in aggregated cell injury (5)

Shenfu injection is a well-known traditional Chinese medicine, which is composed of Radix Ginseng rubra and Radix Aconitum carmichaelii, with ginseng saponin and aconitum alkaloids active ingredients. Previous studies have demonstrated that treatment with Shenfu injection has a curative effect during shock and resuscitation (6), can elevate blood pressure and improve microcirculation to aid in an inflammatory reaction, protect ischemic myocardial cells (7) and prevent acute kidney ischemia-reperfusion injury (8). Furthermore, shenfu injection treatment has also been shown to exhibit good curative effects on transient focal cerebral ischemic injury (9-11), reduce pathological and structural damage in the lungs and liver (12), inactivate anti-oxygen free radical 
xanthine oxidase, improve lung blood flow, adjust the function of immune, system protect vascular endothelial cells, improve hypoxia tolerance and protect from endotoxic shock-induced lung injury (13-15). However, there is a lack of published information investigating the use of Shenfu injection for the treatment of lung ischemia-reperfusion injury.

The present study used a rat model of lung ischemia-reperfusion injury to investigate the role and underlying mechanism of Shenfu injection for the treatment of lung ischemia-reperfusion injury. The following properties were evaluated: Lung wet dry weight (W/D) ratio, tumor necrosis factor- $\alpha$ (TNF- $\alpha$ ) expression levels, myeloperoxidase (MPO), malondialdehyde (MDA) and superoxide dismutase (SOD) activity, and microscopic lung damage.

\section{Materials and methods}

Reagents and kits. Shenfu injection (Z51021920; $100 \mathrm{ml} / \mathrm{ampulla}$; batch no. 100602) was purchased from Ya'an San-Jiu Pharmaceutical Co., Ltd. (Ya'an, China). TNF- $\alpha$ enzyme-linked immunosorbent assay (ELISA; cat no. MTA00B) MDA kits (cat no. A003-1) were provided by Beijing Zhongshan Jinqiao Biotechnology Co., Ltd. (Beijing, China). MPO (cat no. A044) and SOD (cat no. A001-1) kits were produced by the Nanjing Jiancheng Bioengineering Research Institute (Nanjing, China).

Animals. A total of 72 specific pathogen-free healthy male Wistar rats, weighing 250-350 g (age, 60土3 days), were provided by the Lanzhou University Medical College Animal Experiment Center (license no. SCXK 2009-0004). Rats were maintained in $0.063 \mathrm{~m}^{2}$ cages under regulated temperature and humidity (temperature, $19-24^{\circ} \mathrm{C}$; humidity, 55-60\%) with a 12-h dark:light cycle and ad libitum access to water and standard feed.

Drug delivery and groups. Rats were randomly divided into three groups with 24 rats in each group. In the control group, intraperitoneal injection of $0.9 \%$ sodium chloride ( $3 \mathrm{ml} / \mathrm{kg} /$, i.p., $10 \mathrm{sec}$ ) was administered daily for 2 days; after $30 \mathrm{~min}$, the right hilar was divided following thoracotomy without blocking the lung door. In the model group, intraperitoneal injection of $0.9 \%$ sodium chloride $(3 \mathrm{ml} / \mathrm{kg} /$, i.p., $10 \mathrm{sec}$ was administered daily for 2 days; after $30 \mathrm{~min}$, the lung ischemia-reperfusion model was established. In the Shenfu group, the Shenfu injection was diluted 3.7 times with $0.9 \%$ sodium chloride prior to use and intraperitoneal injection of Shenfu ( $3 \mathrm{ml} / \mathrm{kg}$, i.p., $10 \mathrm{sec}$ ) was administered daily for 2 days; after $30 \mathrm{~min}$, the lung ischemia-reperfusion model was established.

Lung ischemia-reperfusion model. An in vivo rat model of lung ischemia-reperfusion was established using the improved Eppinger method $(16,17)$. Anesthesia was induced in rats by an intraperitoneal injection of $10 \%$ chloral hydrate (Lanzhou Chunsheng Chemical Reagent Co., Ltd. (Lanzhou, China; $3 \mathrm{ml} / \mathrm{kg}$ ). Rats were fixed in a supine position on a variable temperature carpet $\left(36 \pm 0.1^{\circ} \mathrm{C}\right)$ and the left chest fur was clipped and disinfected with $75 \%$ alcohol and tincture of iodine. Rats were intubated following tracheotomy, and the lower left pulmonary ligament was divided and ventilated with inhaled air. The following parameters were maintained: PetCO ${ }_{2}, 35-45 \mathrm{mmHg}$; respiratory frequency, 60 times/min; unilateral lung ventilation tidal volume, $8-10 \mathrm{mg} / \mathrm{kg}$; bilateral lung ventilation, $15-20 \mathrm{mg} / \mathrm{kg}$; and inspiration and expiration ratio, 1:2. Following $30 \mathrm{~min}$ ventilation, the left hilus pulmonis was divided using forceps without damaging the artery. Subsequent to the administration of heparin sodium (50 units) to the dorsal vein of the penis was administered and 5 mins later the left lung door was clamped noninvasively. The clamping procedure included the left primary bronchus and the left pulmonary artery and vein. Using a straw, the lung surface was subsequently flushed with a small quantity of cold saline and the wound was covered with sterile warm saline-infiltrated gauze. The vascular clamp was released after $45 \mathrm{~min}$ to allow for reperfusion. At each of the three time points (ischemia $45 \mathrm{~min}$, reperfusion $60 \mathrm{~min}$ and reperfusion $120 \mathrm{~min}$ ), six rats were sacrificed by arterial bleeding. The percentage of alveolar damage (the number of alveoli containing $\geq 2$ red blood cells and white blood cells/200) in the lung following reperfusion was calculated at $120 \mathrm{~min}$.

Bronchial lavage was performed at the reperfusion was performed at the $120 \mathrm{~min}$ time point in another six rats, and the collected broncho-alveolar lavage fluid was stored for cryopreservation at $-70^{\circ} \mathrm{C}$ for the assessment of alveolar damage and total protein. Blood samples collected from the aorta abdominalis were cryopreserved at $-70^{\circ} \mathrm{C}$ for subsequent MDA and SOD analyses. Lung tissue samples were divided into three equal parts, as follows: Upper, a third of the specimen was used to measure the lung W/D ratio; middle, a third of the specimen was fixed in $10 \%$ neutral buffered formalin, embedded in paraffin and subjected to hematoxylin and eosin (HE) staining; and under, a third of the specimen was stored at $-70^{\circ} \mathrm{C}$ for TNF- $\alpha$ and MPO analyses.

W/D ratio. The lung surface was flushed with physiological saline, and the blood was removed. The upper third of the lung tissue and the dry blood on the surface were obtained, weighed, and recorded as wet weight. The dry weight was measured after incubating the samples at $100^{\circ} \mathrm{C}$ for $48 \mathrm{~h}$. Lung $\mathrm{W} / \mathrm{D}$ ratio (\%) was calculated as W/D (100\%).

TNF- $\alpha$ expression levels. In order to assess the levels of TNF- $\alpha$ in the lung homogenates among the groups at three time points, absorbance was determined at $450 \mathrm{~nm}$ wavelength [optical density (OD) value] using a spectrophotometer (Beckman DU-640 Spectrophotometer; Beckman Coulter Commercial Enterprise (China) Co., Ltd.). Preparation of the $10 \%$ lung homogenate with PBS involved specimen and standards for anti-TNF- $\alpha$ monoclonal antibody in an ELISA plate, which was washed. TNF- $\alpha$ expression levels were detected according to the method by Song et al (18) The proportion between TNF- $\alpha$ and OD450 was determined using a standard curve.

MPO activity. Blood vessels and fascia were harvested from the lung tissues and weighed. Using an MPO kit (cat no. A044) produced by the Nanjing Jiancheng Biological Engineering Research Institute (Jiangsu, China) the homogenate medium (number two reagent) was added to lung tissues according to the weight volume ratio of 1:9, homogenized in ice water for $5 \mathrm{~min}$ 
and transformed into $10 \%$ tissue homogenate. Subsequently, $0.9 \mathrm{ml} 10 \%$ tissue homogenate was mixed with $0.1 \mathrm{ml}$ of number three reagent from the MPO kit. Following thorough mixing, the solution was placed in a water bath at $37^{\circ} \mathrm{C}$ for 15 min prior to analysis. The production of $\mathrm{A}$ was measured by colorimetry at $460 \mathrm{~nm}$ wavelength, and MPO activity was calculated as follows: MPO activity $(\mathrm{U} / \mathrm{g})=$ (testing tube OD value - control tube OD value) / 11.3 x sampling weights (g).

SOD activity. Following 10 min blending after the addition of the contents of the SOD kit. The colorimetric analysis was performed using a $1 \mathrm{~cm}$ optical path and $550 \mathrm{~nm}$ wavelength. Distilled water was used for the zero setting. Plasma SOD activity $(\mathrm{NU} / \mathrm{ml})$ was calculated as follows: Plasma SOD activity $=$ (control absorbance - test absorbance $) /$ control absorbance / $0.5 \mathrm{x}$ reaction quantity / sample quantity.

MDA activity. Abdominal aortic blood $(2.0 \mathrm{ml})$ were collected quiescent in an anticoagulant tube for $60 \mathrm{~min}$, and centrifuged at $1,000 \mathrm{x} \mathrm{g}$ for $10 \mathrm{~min}$ to isolate the plasma. MDA was evaluated using a glucosinolate barbituric acid method and calculated as follows: MDA = (test tube absorbance - blank test tube absorbance) / (standard tube absorbance - blank standard tube absorbance) $\mathrm{x}$ standard sample concentration $\mathrm{x}$ number of sample dilution before the test.

Alveolar damage and total protein (TP)/alveolar damage in rats. Using the Belaidi method (3), alveolar white blood cells were counted in ten fields of vision using an MM-800 Nikon microscope (magnification, x200; Nikon Corporation, Tokyo, Japan), and these cells was considered to indicate alveolar leukocyte aggregation. Based on the Muratan method (19), $>200$ alveoli were continuously observed using under x20 and $\mathrm{x} 10$ magnification microscopy. Alveoli that contained $\geq 2$ red blood cells and white blood cells were considered as damaged alveolar and the following equations ere used for calculations: Percentage of alveolar damage $=$ number of damage alveolar $/ 200 \times 100 \%$. Protein content $(\mu \mathrm{g} / \mathrm{g})=[\mathrm{C} \times$ volume after centrifugation $(\mathrm{ml})]$ / sample weight $(\mathrm{g})$. As such, these blood cells were used to identify the quantity of alveolar damage. The concentration of TP in broncho-alveolar lavage fluid was determined using Coomassie brilliant blue staining (Lanzhou Chunsheng Chemical Reagent Co., Ltd.). For HE (Lanzhou Chunsheng Chemical Reagent Co., Ltd.) staining, lung tissues were fixed in formalin for $>24 \mathrm{~h}$ and embedded in paraffin. Samples were then cut $(5 \mu \mathrm{m})$ and subsequently stained with HE.

$H E$ staining. Lung tissues were fixed in $10 \%$ formalin for $>24 \mathrm{~h}$ and embedded in paraffin. Subsequently, they were embedded into wax blocks on a microtome, sliced into 5- $\mu \mathrm{m}$ thick sections, drying was at $45^{\circ} \mathrm{C}$ in a constant temperature oven, the wax was removed with xylene, dehydrated, and finally distilled water was added, and subsequently stained with HE (hematoxylin dye for $5 \mathrm{~min}$, separated in the acid and ammonia water for $30 \mathrm{sec}$, dehydrated in alcohol, stained with eosin dye for 2-3 min. Canada balsam was added dropwise in sections after dehydration with alcohol and treatment with xylene and finally, the cover glass was added. The stains were viewed using a MM-800 Nikon microscope (magnification, x200).
Statistical analysis. Statistical analysis was performed using SPSS 13.0 software (SPSS, Inc., Chicago, IL, USA) via a normal distribution test (Kolmogorov Smirnov test) and homogeneity test for variance (Levene's t-test). Normally distributed data were presented as the mean \pm standard deviation. Between-group comparisons and heterogeneity of variance were analyzed using Student's t-test. $\mathrm{P}<0.05$ was considered to indicate a statistically significant difference.

\section{Results}

Lung W/D ratio. The lung W/D ratio was not significantly different at the three time points in the control group, whereas the lung W/D ratios of rats in the model and Shenfu groups significantly increased with prolonged reperfusion $(\mathrm{P}<0.05)$. The model and Shenfu groups were not significantly different at the ischemia 45 min time point, as compared with the control group, but were significantly increased at the reperfusion $60 \mathrm{~min}$ and reperfusion $120 \mathrm{~min}$ time points $(\mathrm{P}<0.05)$. Lung W/D ratios in the Shenfu group were decreased at reperfusion $60 \mathrm{~min}$ and $120 \mathrm{~min}$, compared with the model group, however, there were no significant differences $(\mathrm{P}>0.05)$. Thus the results revealed that Shenfu injection can reduce the W/D ratios at reperfusion times (Fig. 1).

$T N F-\alpha$. Lung TNF- $\alpha$ expression levels were not significantly different in the control group at each time point. However, TNF- $\alpha$ expression levels were significantly elevated at the reperfusion at the $120 \mathrm{~min}$ time point, compared with the ischemia 45 min time point in the model group and Shenfu injection group $(\mathrm{P}<0.05)$. TNF- $\alpha$ expression levels significantly increased at reperfusion $120 \mathrm{~min}$ in the Shenfu group $(\mathrm{P}<0.05)$, as compared with the ischemia $45 \mathrm{~min}$ and reperfusion 60 min time points. In addition, TNF- $\alpha$ expression levels were significantly elevated at all three time points in the model and Shenfu group, as compared with the control group $(\mathrm{P}<0.05)$. However, TNF- $\alpha$ levels were significantly lower at the reperfusion 120 min time point in the Shenfu group, as compared with the model group $(\mathrm{P}<0.05$; Fig. 2$)$. The present results reveal that Shenfu injection is able to decrease lung TNF- $\alpha$ expression levels at the reperfusion 120 min time point

MPO. No significant differences in serum MPO activity levels were detected among the time points in the control group. MPO activity levels were significantly elevated at the reperfusion $60 \mathrm{~min}$ and reperfusion $120 \mathrm{~min}$ time points, as compared with the ischemia 45 min time point in the model and Shenfu groups $(\mathrm{P}<0.05)$. In addition, MPO activity significantly increased at each time point in the model and Shenfu group, as compared with the control group $(\mathrm{P}<0.05)$. No significant differences in MPO activity levels were detected at the ischemia 45 min time point in the Shenfu group ( $P>0.05)$, but MPO activity levels significantly decreased at the reperfusion $60 \mathrm{~min}$ and reperfusion $120 \mathrm{~min}$ time points, as compared with the model group $(\mathrm{P}<0.05$; Fig. 3). Thus, the present results reveal that Shenfu injection can decrease lung MPO activity levels during reperfusion times.

SOD. No significant differences in SOD activity were detected among the time points in the control group. SOD activity 
significantly decreased at the reperfusion $60 \mathrm{~min}$ and reperfusion 120 min time points, as compared with the activity at ischemia $45 \mathrm{~min}$ in the model group $(\mathrm{P}<0.05)$. In addition, SOD activity was significantly decreased at the reperfusion 60 min time point, as compared with ischemia $45 \mathrm{~min}$ in the Shenfu group $(\mathrm{P}<0.05)$. SOD activity was not significantly different at the reperfusion $60 \mathrm{~min}$ and reperfusion $120 \mathrm{~min}$ time points in the Shenfu group, as compared with the control group. However, SOD activity was significantly reduced at reperfusion $120 \mathrm{~min}$ in the Shenfu group, as compared with the model group $(\mathrm{P}<0.05$; Fig. 4). The results indicate that Shenfu injection can reduce serum SOD activity at the stated reperfusion times.

$M D A$. No significant differences in serum MDA activity were detected among the time points in the control group. MDA activity was significantly higher at the reperfusion $60 \mathrm{~min}$ and reperfusion 120 min time points, as compared with ischemia $45 \mathrm{~min}$ in the model and Shenfu group $(\mathrm{P}<0.05)$. In addition, MDA activity significantly increased at reperfusion $60 \mathrm{~min}$ and reperfusion $120 \mathrm{~min}$ in the model and Shenfu group, as compared with the control group $(\mathrm{P}<0.05)$. MDA activity was not significantly different at ischemia $45 \mathrm{~min}$ in the Shenfu group, as compared with the model group. However, MDA activity was significantly reduced at the reperfusion $60 \mathrm{~min}$ and reperfusion 120 min time points $(\mathrm{P}<0.05$; Fig. 5), as compared with the model group. Thus the results revealed that Shenfu injection is able to reduce serum MDA activity at certain reperfusion times

Alveolar damage and TP. The model and Shenfu groups exhibited varying degrees of alveolar damage and TP concentration increases at the reperfusion 120 min time point, as compared with the control group ( $\mathrm{P}<0.05$; Fig. 6). The degree of alveolar damage in the Shenfu group was significantly decreased, as compared with the model group $(\mathrm{P}<0.05)$. Although no significant changes in TP were detected between the model and Shenfu groups, a marked decrease in TP content was detected in the Shenfu group, as compared with the model group. The results reveal that Shenfu injection can decrease the alveolar damage at the reperfusion 120 min time-point

Light microscopy. The control group exhibited no signs of alveolar damage, with visible organization of alveolar wall integrity, and no interstitial lung edema and neutrophil infiltration. The model group exhibited severely damaged alveolar structures, a large quantity of alveoli, congestive alveolar walls, severe pulmonary interval thickening, wide alveolar spaces, interstitial edema, serous effusion, a large quantity of red blood cells and neutrophil infiltration. Notably, the extent of the damage in the alveolar structure, interstitial neutrophils, and alveolar and interstitial red blood cells was reduced in the Shenfu group, as compared with the control and model groups (Fig. 7). The results indicated that Shenfu injection may relieve the degree of alveolar structure damage.

\section{Discussion}

Emphysema, lung fibrosis, silicosis and bronchial lung expansion are serious end-stage lung diseases that can cause chronic

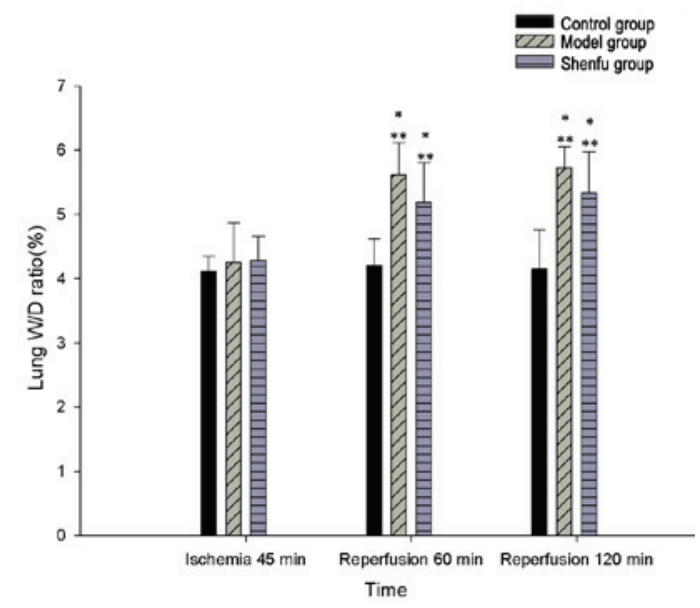

Figure 1. Lung W/D ratio among the groups at three time points. ${ }^{*} \mathrm{P}<0.05$ vs. the control; ${ }^{* *} \mathrm{P}<0.05$ vs. the ischemia time point. W/D, weight/dry weight.

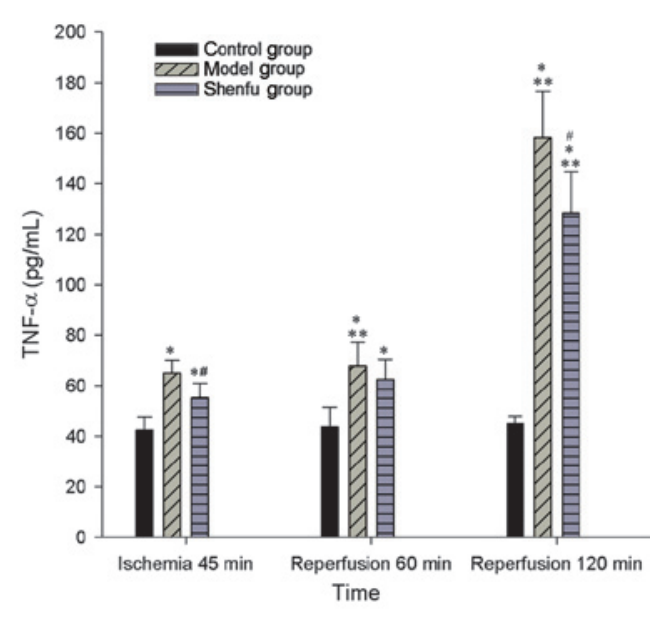

Figure 2. TNF- $\alpha$ levels in lung homogenates among the groups at three time points. ${ }^{*} \mathrm{P}<0.05$ vs. the control group; ${ }^{* *} \mathrm{P}<0.05$ vs. the ischemia time point; ${ }^{\#} \mathrm{P}<0.05$ vs. the model group. TNF- $\alpha$, tumor necrosis factor- $\alpha$.

respiratory function failure and result in patients requiring a breathing machine. To date, lung transplantation remains the only effective treatment method for end-stage lung disease (20). Lung transplantations have been performed for $>30$ years in China and statistical analyses have demonstrated that one-year patient survival percentage is $70 \%$. Lung transplant failure is predominantly a result of lung ischemia-reperfusion injury, which has a mortality rate of $25 \%(21,22)$. Ischemia-reperfusion is also a risk factor for chronic rejection following transplantation, which affects the long-term survival of patients.

The mechanism underlying lung ischemia-reperfusion injury is yet to be fully elucidated; although increased pulmonary vascular permeability and dysfunction, and further lung damage following lung ischemia-reperfusion are thought to be associated. Microscopy has been used to observe damaged endothelial cells following the apoptosis and necrosis of pulmonary capillary and alveolar epithelial cells following ischemia-reperfusion injury $(1,2)$. To date, three theories have been proposed to explain the mechanism underlying lung ischemia-reperfusion injury. The neutrophil theory indicates that numerous chemical chemotaxes, including 


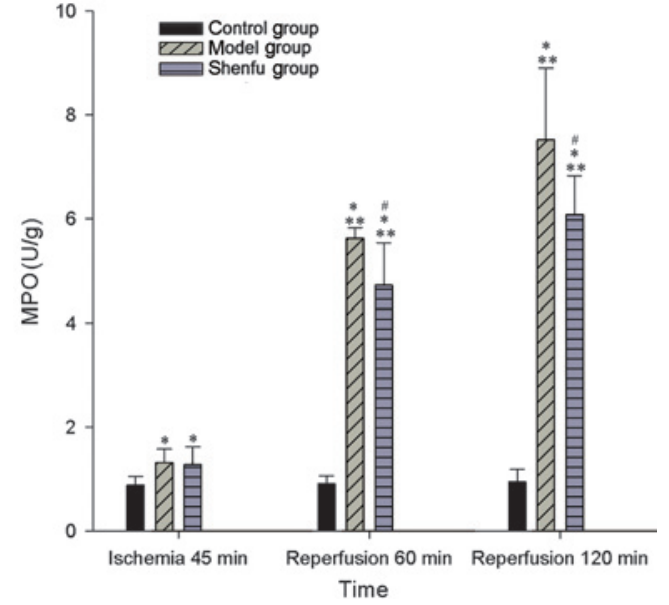

Figure 3. MPO activity levels in lung tissue among the groups at three time points. ${ }^{*} \mathrm{P}<0.05$ vs. the control group; ${ }^{* *} \mathrm{P}<0.05$ vs. the ischemia time point; ${ }^{\#} \mathrm{P}<0.05$ vs. the model group. MPO, myeloperoxidase.

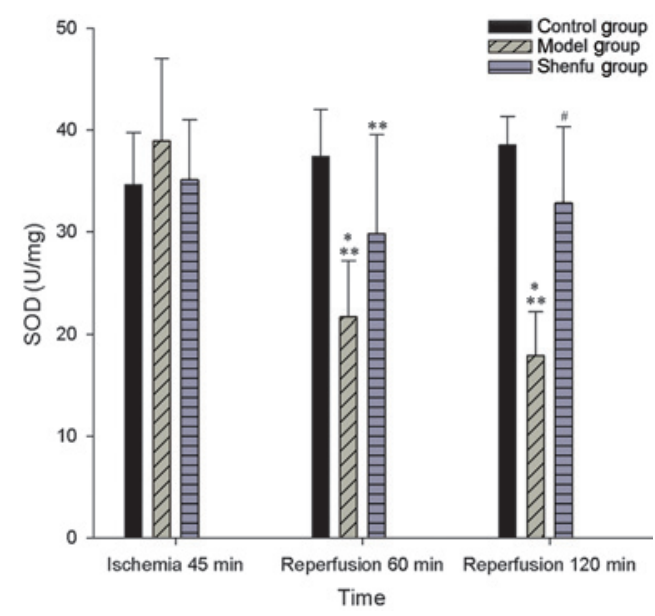

Figure 4. SOD activity levels in blood samples among the groups at three time points. ${ }^{*} \mathrm{P}<0.05$ vs. the control group; ${ }^{* *} \mathrm{P}<0.05$ vs. the ischemia time point; ${ }^{*} \mathrm{P}<0.05$ vs. the model group. $\mathrm{SOD}$, superoxide dismutase.

leukotriene and TNF, are released into the blood following ischemia-reperfusion. The free radical theory states that when ischemia occurs in pulmonary capillary endothelial cells, xanthine dehydrogenase is converted into xanthine oxidase, which exists in the endothelium. Adenosine is degraded by xanthine oxidase and produces a large quantity of oxygen free radicals following reperfusion to mediate lipid peroxidation, inhibit protein function, and induce endothelial cell membrane damage, endothelial edema and jam capillary (23). Free radicals are also capable of promoting inflammatory factors and neutrophil aggregation and activation, thereby resulting in lung tissue damage and the decline of lung function (24). The calcium overload theory states that following lung ischemia, the adenosine triphosphate concentration in cells decreases, the activity of sodium pumps decreases, intracellular $\mathrm{Na}^{+}$ion concentration increases and cellular edema occurs. Following reperfusion, the high intracellular $\mathrm{Na}^{+}$ion content activates $\mathrm{Na}^{+} / \mathrm{Ca}^{2+}$ exchange proteins, and $\mathrm{Na}^{+}$is extracellularly transported. A large quantity of $\mathrm{Ca}^{2+}$ ions simultaneously enter the cells, thereby causing calcium overload. Subsequently,

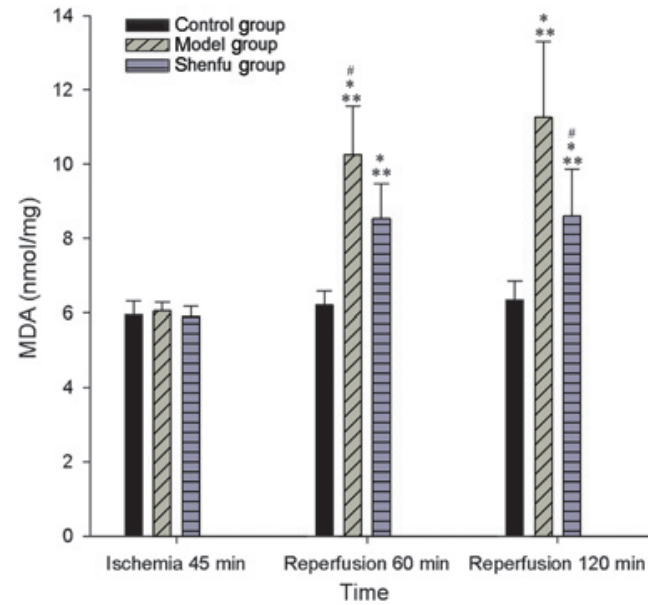

Figure 5. MDA activity levels in blood samples among the groups at three time points. ${ }^{*} \mathrm{P}<0.05$ vs. the control group; ${ }^{* *} \mathrm{P}<0.05$ vs. the ischemia time point; ${ }^{\prime} \mathrm{P}<0.05$ vs. the model group. MDA, malondialdehyde.

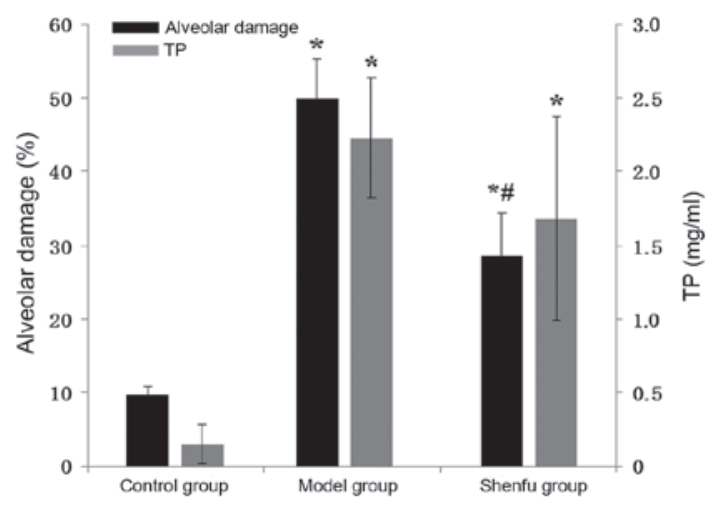

Figure 6. Alveolar damage and TP after reperfusion for $120 \mathrm{~min}$ among the groups. ${ }^{*} \mathrm{P}<0.05$ vs. the control group; ${ }^{\text {}} \mathrm{P}<0.05$ vs. the model group. TP, total protein.

the damaged cell membrane, mitochondria, and sarcoplasm retina increase the permeability of the membrane, resulting in endothelial edema (25). This condition may interfere with the oxidative phosphorylation of the mitochondria, thereby reducing ATP production, promoting free radical generation, and damaging lung tissues (23). A number of drugs prescribed to treat moderate or reduced ischemia-reperfusion injury are available in clinics, including: SOD and calcium antagonists, auxiliary medication with prostaglandin to dilate blood vessels, and sodium nitrate to improve arterial blood oxygenation (26).

Based on the suggested mechanisms underlying lung ischemia-reperfusion injury, targeted therapy drugs and data from animal experiments, clinical experience, and related pervious studies, it can be speculated that Shenfu injection may reduce lung ischemia-reperfusion injury. As studies investigating Shenfu injection and its effect on lung ischemia-reperfusion injury are lacking $(12,27,28)$, the present study aimed to examine the role of Shenfu injection in a rat model of lung ischemia-reperfusion to determine the mechanisms underlying its protective effects.

Shenfu injection originates from the traditional medicine Shenfu decoction, which is extracted from red ginseng and black aconitum carmichaelii. The primary ingredients of Shenfu 
A

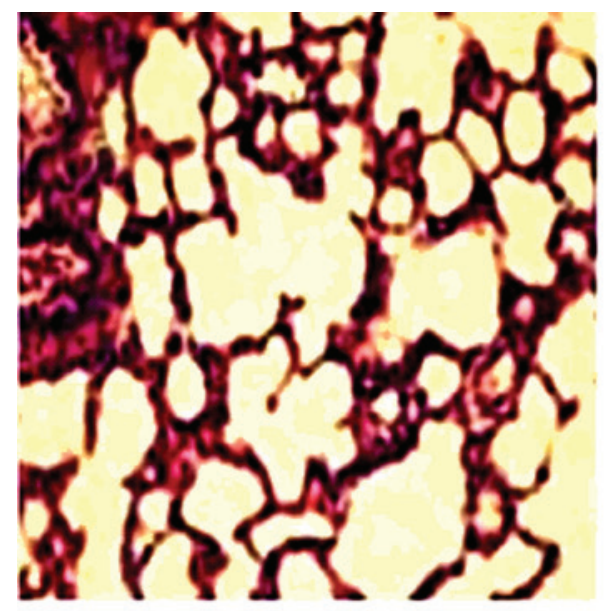

B

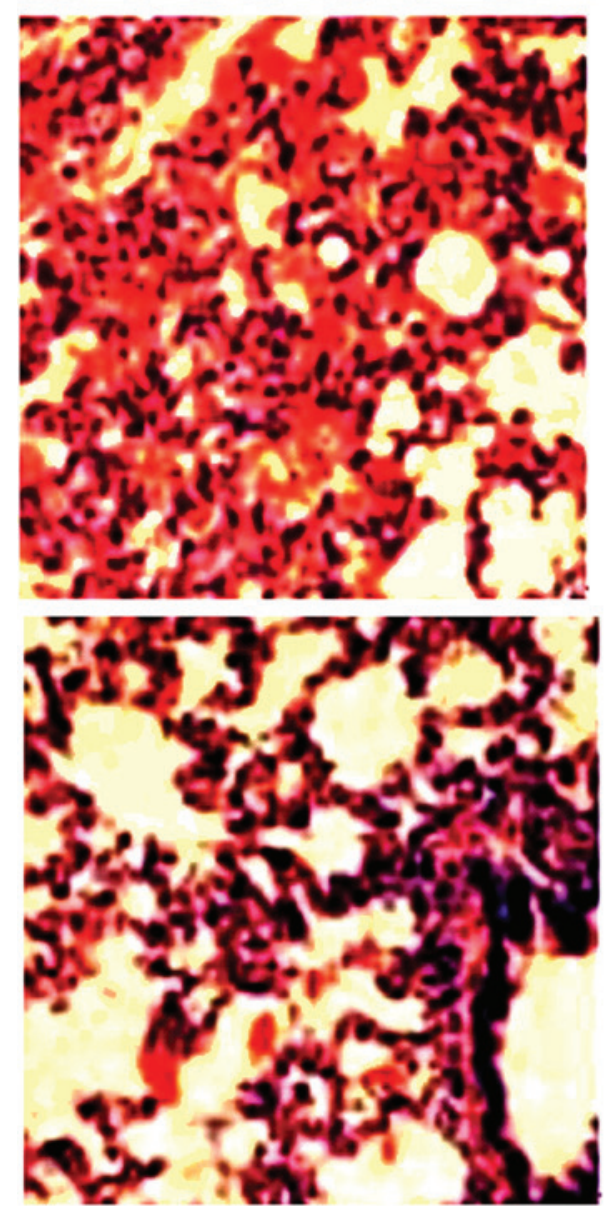

Figure 7. Hematoxylin and eosin staining of (A) the control, (B) model and (C) Shenfu groups. No signs of alveolar damage were detected in the control group; whereas the model group exhibited severely damaged alveolar structures, serous effusion, a large quantity of red blood cells and neutrophil infiltration. The extent of the damage to the alveolar structure, interstitial neutrophils, and alveolar and interstitial red blood cells was lower in the Shenfu group compared with the control and model groups.

injection include ginseng saponin Rb1 ( $\geq 0.5 \mathrm{~g} / \mathrm{l})$ and aconitum alkaloids $(\leq 0.1 \mathrm{~g} / \mathrm{Ul})$. Previous studies have demonstrated that ginseng saponins exert protective effects against myocardial ischemia-reperfusion injury (29). Furthermore, Shenfu injection is capable of increasing the activity of SOD, decreasing MDA levels, removing oxygen free radicals, protecting against lipid peroxidation, antagonizing $\mathrm{Ca}^{2+}$ and producing a glucocorticoid-like effect (25). It has also been demonstrated that Shenfu injection can stabilize the cell membrane, reduce myocardial enzyme release and protect ischemic myocardial cells (7). A previous study, which used an adult rabbit model of acute kidney ischemia-reperfusion, demonstrated that Shenfu injection can protect SOD activity. SOD is an endogenous oxygen free radical scavenger that directly inactivates oxygen free radicals, increases nitric oxide, inhibits leukocyte adhesion and $\mathrm{Na}^{+}$in-flow, and prevents acute ischemia-reperfusion injury (8). Zheng et al (7) demonstrated that different doses of intraperitoneal Shenfu injection $(5,10$ and $20 \mathrm{ml} / \mathrm{kg}$ ) have a dose-dependent protective effect on transient focal cerebral ischemia. Previous clinical studies have demonstrated that Shenfu injection exhibits good curative effects on bronchial asthma and chronic obstructive pulmonary disease $(6,8,11)$. Furthermore, modern pharmacological studies have confirmed that Shenfu injection is capable of inhibiting the activation of nuclear transcription factor $\kappa \mathrm{B}$ and decreasing the expression levels of TNF- $\alpha$ and interleukin (IL)-6, thereby reducing pathological damage in the lungs and liver and protecting the structures of these organs (12). Shenfu injection directly inactivates the anti-oxygen free radical xanthine oxidase, improve lung blood flow, adjust immune function (30). Shenfu injection also protects vascular endothelial cells, functions as an anti-inflammatory and anti-endotoxin compound, improves hypoxia tolerance, and protects against lung injury induced by endotoxic shock (13-15) Therefore, we hypothesize that Shenfu injection at $10 \mathrm{ml} / \mathrm{kg}$ dosage can exert protective effects on cells when applied prior to lung ischemia-reperfusion injury.

Following lung ischemia-reperfusion, blood flow and ventilation rapidly increase, oxygen levels in lung tissues increase sharply, and oxygen free radicals that attack unsaturated fatty acids in biological membranes are released; thus, lipid peroxidation occurs and forms lipid peroxides, which induce fluid loss from the cell membrane and tissue edema, increase permeability and aggravate lung injury (31-33). Lung W/D ratio is an index of lung water content which is used to assess the degree of lung edema and reflect the degree of lung injury. In the present study, the rat lung W/D ratio significantly increased at the ischemia $45 \mathrm{~min}$ and reperfusion 120 min time points, indicating the occurrence of high lung water content and edema following lung ischemia-reperfusion. The W/D ratio significantly decreased in the Shenfu injection group, as compared with the model group, indicating that Shenfu injection can inhibit edema and reduce lung injury. This finding implied that Shenfu injection may increase the blood flow in the ischemia/non-ischemia area and stabilize the hemodynamics. Hanci et al (34) have identified that the effects of Shenfu injection are correlated with its dosage.

TNF, which is an important pre-inflammatory factor, exists in two forms, namely TNF- $\alpha$ and TNF- $\beta$. TNF- $\alpha$ is secreted by activated monocytes/macrophages and has a molecular weight of $17 \mathrm{kDa}$. TNF- $\alpha$ promotes immunity and inflammatory reactions. During ischemia-reperfusion, TNF- $\alpha$ concentration rapidly increases and exhibits higher sensitivity than other cytokines (23). TNF- $\alpha$ can activate and promote humoral inflammatory reactions which may induce a series of pathological responses in the body, including the degranulation of neutrophils, and the expression of CD11b/CD18 adhesion molecules. TNF- $\alpha$ also triggers the adhesion of CD11b/CD18 on vascular endothelial cells, amplifies low stimulation reaction, 
damages vascular endothelial cells, increases vascular permeability, reduces circulation resistance, induces the release of other humoral inflammatory factors, including IL-8, leukotriene and platelet activating factor, and increases the phagocytosis of macrophages $(35,36)$. Therefore, TNF- $\alpha$ can be used as an inflammatory indicator in the body and TNF- $\alpha$ expression levels are associated with the degree of damage.

SOD, which is a primary oxidase and super-organism oxygen free radical scavenger, has been demonstrated to reflect the ability of the body to remove free radicals. SOD disproportionates the lipid peroxide (as MDA) for hydrogen peroxide and water, thereby protecting cells from damage. Previous animal studies have demonstrated that SOD levels decrease in response to the large quantity of oxygen free radicals following reperfusion; such a decrease in SOD concentration damages the structure and function of the endothelial cells in lung tissues $(37,38)$. The current study demonstrated that Shenfu injection may reduce SOD levels in plasma following reperfusion.

MDA, which is a metabolic product of lipid peroxidation, is important in lipid peroxidation of membrane lipid failure, and can reflect the degree of lipid peroxidation in the body as an indirect index of the degree of lung reperfusion injury (39). The present study demonstrated that high levels of MDA were expressed during lung injury in rats following lung ischemia-reperfusion, which confirmed that the present rat model of lung ischemia-reperfusion was successful. The results demonstrated that Shenfu injection improved resistance to oxygen free radical damage and reduced lung ischemia-reperfusion injury by inhibiting the oxidative stress reaction.

In conclusion, the results of the present study suggested that Shenfu injection may reduce the lung W/D ratio and the concentration of TNF- $\alpha$ and MPO in rats. Furthermore, Shenfu injection also reduced plasma SOD and MDA expression levels and TP content in broncho-alveolar lavage fluid. Therefore, Shenfu injection may reduce the physical injury of the lung, and to an extent protects against ischemia-reperfusion injury. As a result, we recommend that further investigation into the clinical application of Shenfu injection prior to ischemia-reperfusion injury is performed.

\section{Acknowledgements}

The present study was supported by the Hospital Foundation of the First Hospital of Lanzhou University (grant no. ldyyynlc201206).

\section{References}

1. Yang CL, Tsai PS and Huang CJ: Effects of dexmedetomidine on regulating pulmonary inflammation in a rat model of ventilator-induced lung injury. Acta Anaesthesiol Taiwan 46: 151-159, 2008.

2. Harada M, Oto T, Otani S and Miyoshi K: A neutrophil elastase inhibitor improves lung function during ex vivo lung perfusion. Gen Thorac Cardiovasc Surg 63: 645-651, 2015.

3. Belaïdi E, Béguin PC, Ribuot C and Godin-Ribuot D: Hypoxic preconditioning: Role of transcription factor HIF-1alpha. Ann Cardiol Angeiol (Paris) 55: 70-73, 2006 (In French).

4. Kurt A, Tumkaya L, Kalkan Y, Turut H, Cure MC, Cure E, Sehitoglu I, Bilgin $\mathrm{H}$ and Usta M: Is adalimumab protective in ischemia-reperfusion injury in lung? Iran J Basic Med Sci 18 1093-1099, 2015.
5. Zhang XJ, Song L, Zhou ZG and Wang XM: Effect of shenfu injection on gastrointestinal microcirculation in rabbits after myocardial ischemia-reperfusion injury.World J Gastroenterol 12: 4389-4391,2006.

6. Dong G and Shen B: Clinical effect of shenfu injection in the sepsis bundle treatment of septic shock. Zhong Guo Zhong Yi Ji Zheng 23: 944-945, 2014 (In Chinese).

7. Zheng SY, Sun J, Zhao X and Xu JG: Protective effect of shen-fu on myocardial ischemia-reperfusion injury in rats. Am J Chin Med 32: 209-220, 2004.

8. Yang SL, Feng ZQ, Wu LS and Li LH: Shenfu injection prevents kidney from acute ischemia-reperfusion injury in rabbits. Zhong Guo Bing Li Sheng Li Za Zhi 19: 353-356, 2003 (In Chinese).

9. Long SQ, Liao GY, He WF, Wang B, Deng H Zhang HB, Chai XS, Cau JZ and Wu WY: Influence of shenfu injection on the quality of life of lung cancer patients receiving chemotherapy. Nan Fang Yi Ke Da Xue Xue Bao 31: 2090-2092, 2011 (In Chinese).

10. Huang F, Chen X and Zhu J: Clinical study on the effects of shenfu injection in children with bronchial asthma. Zhong Guo Xian Dai Yi Sheng 52: 70-72, 2014 (In Chinese).

11. Fang M: Clinical observation on treatment of 32 cases of chronic obstructive lung disease with Chinese medicine combined with Western medicine. Jiang Su Zhong Yi Yao 39: 22-23, 2007 (In Chinese).

12. Wang J, Qiao LF and Yang GT: Role of Shenfu Injection in rats with systemic inflammatory response syndrome. Chin J Integr Med 14:51-55, 2008

13. Hu G, Liu XY, Xia ZY and Xiong GX: The effect of shenfu injection on the expression of NF- $\mathrm{B}$, ICAM-1, TNF- $\alpha$ after ischemia-reperfusion of intestinal mucosa in rats. Tong Ji Da Xue Xue Bao 24: 381-384, 2003 (In Chinese).

14. Yun QC: Effect of combined methylprednisolone with shenfu injection on the treatment of children systemic inflammatory response syndrome. Zhong Guo Zhong Xi Yi Jie He Ji Jiu Za Zhi 9: 155-156, 2002 (In Chinese).

15. Zhang Q, Li CS, Wang S and Gu W: Effects of Chinese medicine Shen-Fu injection on the expression of inflammatory cytokines and complements during post-resuscitation immune dysfunction in a porcine model. Chin J Integr Med 22: 101-109, 2016.

16. Chai J, Wang DW and Shen J: Effect of sevoflurane on pulmonary oxygen free radicals in ischemia-reperfusion rats. Shi Yong Yao Wu Yu Lin Chuang 12: 156-159, 2009 (In Chinese).

17. Du JZ and Dong BR: The effect of atorvastatin on pulmonary ischemia-reperfusion injury in rats. Zhong Guo Ji Ceng Yi Yao 13: 2027-2028, 2006 (In Chinese).

18. Song J, Jiang M, Qi G, Xie Y, Wang H, Tian Y, Qu J, Zhang X and $\mathrm{Li} \mathrm{H}$ : Effect of airway humidification on lung injury induced by mechanical ventilation. Zhonghua Wei Zhong Bing Ji Jiu Yi Xue 26: 884-889, 2014 (In Chinese).

19. Dawn B and Bolli R: HO-1 induction by HIF-1: A new mechanism for delayed cardioprotection? Am J Physiol Heart Circ Physiol 289: H522-H524, 2005

20. Laubach VE and Sharma AK: Mechanisms of lung ischemia-reperfusion injury. Curr Opin Organ Transplant 21: 246-252, 2016.

21. Lehmann S, Barten MJ, Topf C, Garbade J, Dhein S, Mohr FW and Bittner HB: Donor type impact on ischemia-reperfusion injury after lung transplantation. Ann Thorac Surg 93: 913-919, discussion 919-920, 2012.

22. Zhang Z,Shen H,Qin HD,Ma MZ and Xu Y: Protective effect of $\mathrm{N}$-acetylcysteine against pneumocyte apoptosis during ischemia/reperfusion injury of lung in rats. Zhongguo Wei Zhong Bing Ji Jiu Yi Xue 24: 111-115,2012 (In Chinese).

23. Reyes YA, Shimoyama T, Akamatsu H and Sunamori M: MCI-186 (edaravone), a free radical scavenger, attenuates ischemia-reperfusion injury and activation of phospholipase $\mathrm{A}(2)$ in an isolated rat lung model after $18 \mathrm{~h}$ of cold preservation. Eur J Cardiothorac Surg 29: 304-311, 2006.

24. Ferrari RS and Andrade CF: Oxidative Stress and Lung Ischemia-Reperfusion Injury. Oxid Med Cell Longev 2015: 590987, 2015.

25. Wu Y, Xia ZY, Meng QT, Zhu J, Lei S, Xu J and Dou J: Shen-Fu injection preconditioning inhibits myocardial ischemia-reperfusion injury in diabetic rats: Activation of eNOS via the PI3K/Akt pathway. J Biomed Biotechnol 2011: 384627, 2011.

26. Jin WY, Sun W, Zhang YZ, Li HY and Chen MZ: Effect of benidipine on plasma level of calcitonin gene-related peptide in essential hypertension. Zhonghua Nei Ke Za Zhi 43: 747-749, 2004 (In Chinese). 
27. Ma RH, Li Y and Wang YX: Postconditioning with shenfu injection protects against pulmonary ischemia/reperfusion injury in rats MA. Zhong Guo Yao Wu Yu Lin Chuang 13: 437-439, 2013 (In Chinese)

28. Xia ZY, Meng QT, Zhang F and Chen XD: Effect of shenfu injection (ginesenoside and aconite alkaloid) on the apoptosis of intestinal mucosal epithelial cells and its mechanism during ischemia-reperfusion in rats. Zhong Hua Chuang Shang Za Zhi 7: 363-367, 2004 (In Chinese).

29. Wang ZF: The protective of Shenfu injections on hemodynamics and myocardial enzyme after myocardial ischemia/reperfusion injury. Zhongguo Ying Yong Sheng Li Xue Za Zhi 27: 155-157, 2011 (In Chinese).

30. Gu W, Hou X, Zhou H and Li C: Protective effect of shen-fu injection on neuronal mitochondrial function in a porcine model of prolonged cardiac arrest. Evid Based Complement Alternat Med 2014: 523847, 2014.

31. Christie JD, Edward LB, Aurora P, Dobbels F, Kirk R, Rahmel AO, Stehlik J, Taylor DO, Kucheryavaya AY and Hertz MI: The Registry of the International Society for Heart and Lung Transplantation: Twenty-sixth Official Adult Lung and Heart-Lung Transplantation Report - 2009. J Heart Lung Transplant 28: 1031-1049, 2009.

32. Ng CS, Wan S, Yim AP and Arifi AA: Pulmonary dysfunction after cardiac surgery. Chest 121: 1269-1277, 2002.

33. Friedrich I, Spillner J, Lu EX, Bartling B, Barnscheid M, Sablotzki A, Schade U, Reidemeister JC, Silber RE, Gunther A and Borgermann $\mathrm{J}$ : Ischemic pre-conditioning of 5 minutes but not of 10 minutes improves lung function after warm ischemia in a canine model. J Heart Lung Transplant 20: 985-995, 2001.
34. Hanci V, Yurdakan G, Yurtlu S, Turan IO and Sipahi EY: Protective effect of dexmedetomidine in a rat model of $\alpha$-naphthylthiourea-induced acute lung injury. J Surg Res 178: 424-430, 2012.

35. Kesici S, Turkmen UA, Kesici U, Altan A and Pulat E: Effects of enteral and parenteral glutamine on intestinal mucosa and on levels of blood glutamine, tumor necrosis factor-alpha, and interleukin-10 in an experimental sepsis model. Saudi Med J 33: 262-271, 2012.

36. Fu PK, Wu CL, Tsai TH and Hsieh CL: Anti-inflammatory and anticoagulative effects of paeonol on LPS-induced acute lung injury in rats. Evid Based Complement Alternat Med 2012: 22454687, 2012.

37. Inci I, Dutly A, Inci D, Boehler A and Weder W: Recipient treatment with trimetazidine improves graft function and protects energy status after lung transplantation. J Heart Lung Transplant 20: 1115-1122, 2001.

38. Li YC and Jiang Z: Ulinastatin, a human protease inhibitor attenuates ischemia-reperfusion lung injury. Fu Dan Xue Bao Yi Xue Ban Bian Ji Wei Yuan Hui 31: 182-186, 2004 (In Chinese).

39. Xu M, Wen XH, Chen SP, An XX and Xu HY: Addition of ulinastatin to preservation solution promotes protection against ischemia-reperfusion injury in rabbit lung. Chin Med J (Engl) 124: 2179-2183, 2011. 